\title{
THE EVO 2+4-ROLL BAR REDUCING AND SIZING MILL*
}

\author{
Alberto Lainati $^{1}$ \\ Luigi Giacomini²
}

\begin{abstract}
The Bar Reducing and Sizing Technology has a core function in the value-chain for the production of rolled SBQ (Special Bar Quality) products. The EVO 2+4-roll is based on the compact combination of a 2-roll high-reduction module followed by a 4roll low-reduction module for precise sizing. The EVO 2+4-roll mill, allows to gain significant technical and economical advantages in the hot- and post-rolling process routes: dimensional and surface accuracy, quick adaptability to processing variances and to small lots requirements, superior technological features of as-rolled product, competitive capex and opex. The EVO $2+4$ unit has a very compact design, which makes it suitable both for new mills and upgrade applications.
\end{abstract}

Keywords: SBQ; Accuracy; Free-sizing; Zero spread. 


\section{INTRODUCTION}

The market of Special-Bar-Quality long products (SBQ) is very vital and promising, both in established and in growing markets. Automotive and high-end mechanic applications are the main drivers for the demand of the SBQ products, in a premium market for very qualified producers exposed anyhow to an increasing price pressure. Technical measures and economical returns need to be balanced among the two main players of the SBQ production value-chain, that is hot and post-rolling producers.

Despite the constant progression of the rolling process in terms of size accuracy, surface finishing, workability of as-rolled product (i.e. metallurgical and mechanical features) and yield, the value-chain has still a significant room for optimization in terms of flexibility, efficiency and stability of quality. Many and varied demands come from the producers and from the end-users of the SBQ products, ranging from technological factors, to operational and economical ones.

The needs are in some extent uniform but sometimes also in contrast, thus making difficult to select a unique production technology able to satisfy all of them.

The traditional Bar Reducing and Sizing Mills, both based on 2-roll or 3-roll groups of rolling stands, has demonstrated to be a central tool to answer a good part of the technological and economical needs, but still not the absolute answer. It is detected in fact that, despite the process improvements obtained by rolling with traditional reducing and sizing mills, the typical "loss" of material along the production chain is still surely more than $10 \%$, with a good part of the inefficiency rate related to the difficulty to maintain a stable quality of the rolled bar.

Further to this material loss, also the productivity loss due to excessive mill stoppages plus unbalanced investment and high operational cost can hinder the competitiveness of a producer.

Looking at the upstream part of the SBQ production chain, it is known that the combined requirements of thermo-mechanical rolling (low temperature rolling, high reduction rate and metallurgical uniformity) with the high shaping/finishing accuracy of the rolled bar, require a specific group of stands - named reducing and sizing mill - arranged in particular configurations (i.e. 2-roll $\mathrm{H}-\mathrm{V}$ or 3-roll $\mathrm{Y}-\lambda$ or 4-roll $\mathrm{X}$ ) and number of units (i.e. 2-roll group with $3 \mathrm{H}-\mathrm{V}-\mathrm{H}$ stands or $4 \mathrm{H}-\mathrm{V}-\mathrm{H}-\mathrm{V}$, 3-roll group with 4 or 5 stands, 4 -roll with 1 or 2 stands).

The technical requirements can be basically satisfied both by 2-roll and 3-roll reducing sizing groups, even with different levels of operational performance and consistency; the 4-roll technique has instead a quite limited application just for finishing sizing and cannot cover all the requirements.

The 2-roll groups can grant accurate rolling finishing at higher rolling reduction rate with a better and deeper strain penetration in the bar thus granting absolute metallurgical homogeneity through the bar section, but has indeed a less efficient shaping with higher material spread and a lower adaptability to the free-sizing requirements.

The 3-roll groups can grant accurate and efficient rolling with reduced material spread but apply a less deep and distributed strain penetration, thus resulting in a less homogeneous metallurgical structure. They have a wide possibility of application of the free-sizing mode even if, at the extremes of the sizes-family in the free-sizing mode, the size accuracy is somehow jeopardized. 
In 2011 Primetals started an R\&D project for an evolved concept of reducing and sizing group based on a unique combination of the 2-roll and 4-roll processing techniques.

\section{THE EVO REDUCING AND SIZING MILL FOR THE NEEDS OF THE MODERN MARKET}

The challenge was to conceive an innovative mechatronic processing line able to roll a wide mix of SBQ/Special grades bars with superior and consistent as-rolled quality (size tolerance + surface finishing + metallurgical texture) with reduced transformation cost and energy consumption, labor-free operations, high mill availability and extreme flexibility in production planning.

The result is called EVO 2+4-roll Reducing and Sizing Mill, a modular machine-tool concept which offers satisfactory answers to the main market demands, granting advantages both to the hot-rolling producers and to the post-rolling end-users. This patented technology (Figure 1) is based on an high-reduction oval-round shaping sequence (reduction ranging from 20 to $55 \%$ ) followed by low-reduction multi-roll finishing round-round-round passes (with total reduction ranging from 4 to 25\%) featuring the so called zero-spread rolling. The rolling temperatures, specifically low temperature prescriptions to exploit the metallurgical benefits, is also part of the patented process.

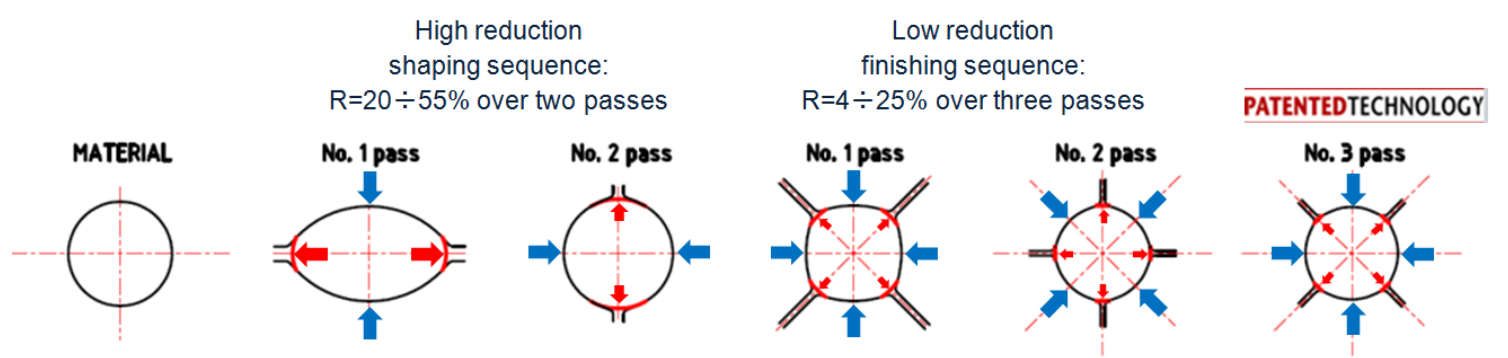

Figure 1. 2+4-roll patented process

The EVO 2+4-roll mill comprises a group of five stands: two 2-roll stands followed by three 4-roll stands. The two 2-roll stands are arranged in an "X" configuration, while each of the following three 4-roll stands have a $45^{\circ} \mathrm{X}+\mathrm{X}$ orientation. The two modules of stands are independent and driven by individual motors, one for each 2-roll stand, two for each 4-roll stand.

The group is extremely compact thus reducing the charges associated to layout footprint. The group can therefore be implemented also as brown-field installation in existing mills which need to upgrade the production quality; it requires less invasive foundation works compared to other existing technologies, the figure 2 shows the modular arrangement and the overall dimensions for an EVO 2+4-roll mill suitable for the rolling of rounds up to $100 \mathrm{~mm}$ diameter. Civil works are very simple and the access to the machine at working level is very easy thanks to lean and underneath design of the transmission system. 

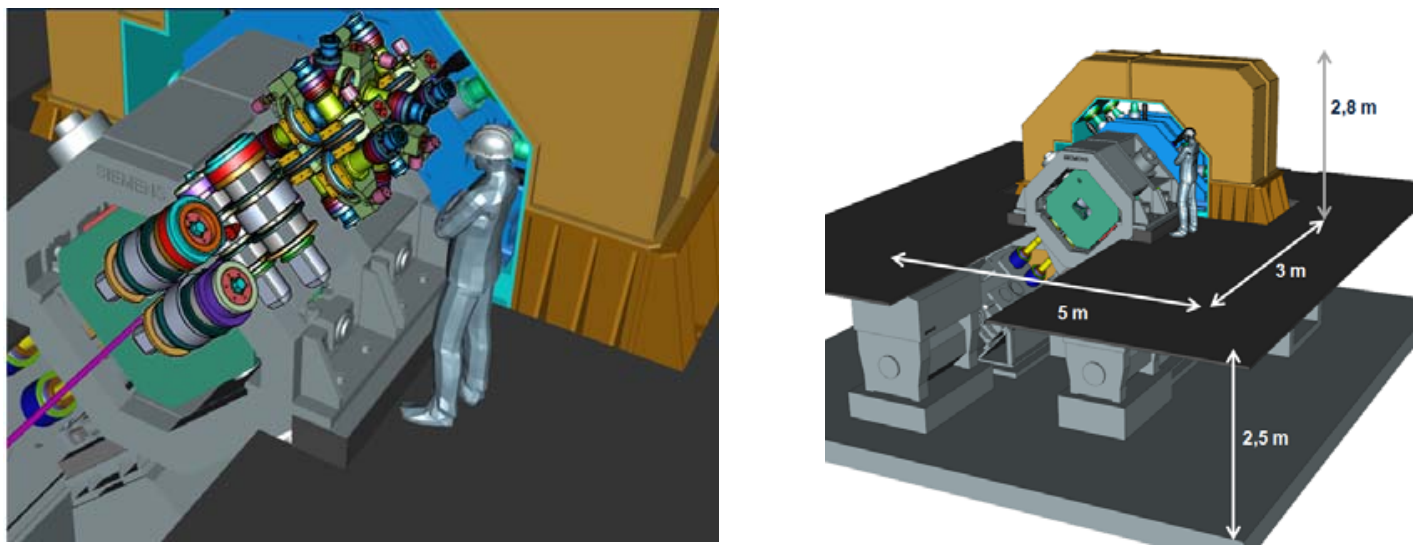

Figure 2. EVO 2+4-roll modules and installation footprint

\section{HIGHLIGHTS OF PROCESS DESIGN}

The principal requirements of an efficient rolling train to produce SBQ products are known to be the following:

- large mix of rollable products, both as shapes (i.e. rounds, hexagons, squares, flats) and sizes range

- single family rolling in the upstream train to reduce operational downtime for change of sequence as well as the roll-shop times and inventory

- precision rolling with tolerances equal or better that 1/6 DIN according to International Standards

- free-sizing rolling to be able to roll a family of sizes around a nominal product with the same set of grooves after simple gap adjustment. This is typically necessary when every size has to be rolled in small lots, as frequently happens when producing high grades of steel

- full thermo-mechanical rolling, combining high reduction with low temperature to grant optimized and uniform metallurgical texture (i.e. grain size >8 ASTM, with maximum 1 ASTM of difference within the bar section)

- smooth defect-free surface finishing of bars

While having reached an elevated level of performance, the conventional 2-roll and 3-roll reducing and sizing technologies cannot match in total the above requirements and imply some compromises of application, sometimes acceptable but often penalizing the economical result of the producer.

Design strength and flexibility of use make the EVO 2+4-roll Mill able to comply with all the requirements. Specifically, the high rolling reduction applicable by the two 2roll stands, allows the single-family rolling and the thermo-mechanical rolling extended up to large sizes (i.e. round $65 \mathrm{~mm}$ and more), while the zero-spread rolling in the compact 4-roll stands grants a wider free-size rolling with accurate size tolerance also at the extremes of the sizes family, always maintaining a uniform metallurgical texture.

Single-family and free-size rolling are important to improve mill utilization, its flexibility of operation, and the equipment inventory management (rolls, guides), bringing an overall reduction of operating costs. In SBQ markets, even very small lots of nonstandard sizes may be economically produced with a minimum impact on mill utilization and within tolerances. For example, an EVO 2+4-roll mill is capable to process any size of round ranging from 14 to $100 \mathrm{~mm}$ in a single-family pass design, 
with no changes to the upward mill, and free-size it into about seventy finished calibrated diameters using only 14 sets of rolls (Figure 3 ).

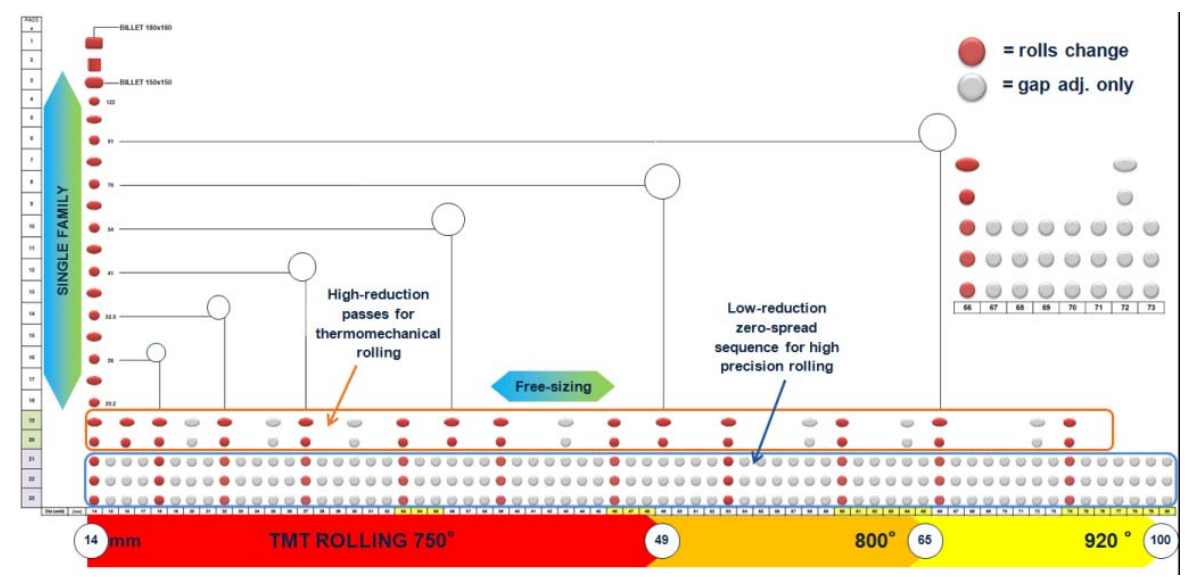

Figure 2. Typical pass schedule with EVO 2+4-roll mill

The zero-spread rolling is a peculiar features of the 4-roll pass shaping and gives some great advantages (Figure 4):

- to reduce the energy consumption for rolling (up to minus 15\%) because all reduction goes in elongation

- to accept a larger difference of area of the entry stock, as typically happens when rolling in sequence many steel grades of the same size

- to reduce the roll wear due to the limited speed difference in the contact points

- to avoid local overheating to the bar under rolling due to the uniform strain distribution, thus assuring a very homogeneous grain size in the bar section

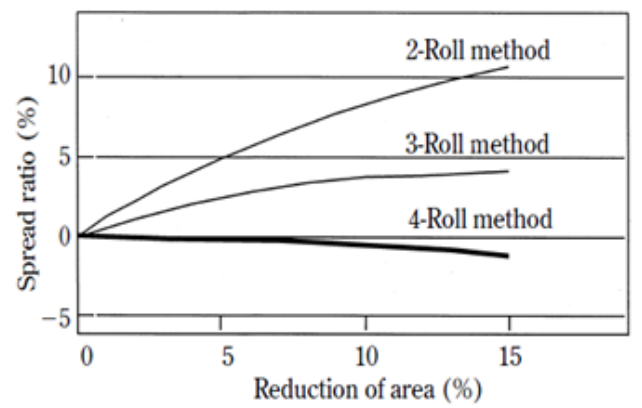

Figure 4. Spread ratio comparison among 2-, 3- and 4-roll

With zero-spread and the under-load gap adjustment possibility, the 4-rolls method can compensate automatically important cross-sectional variations of the stock entering the reducing and sizing sequence attributable to different spread behavior of steel grades, to head/tail effect and to instability of bar temperature profile. The acceptable variation of area for the entry stock can be even higher than $20 \%$, then gradually recovered in the three 4-roll passes by under-load automatic gap compensation. The pass wear is reduced as well by $20 \%$ compared to 3 -roll pass due to the nearly flat roll profile (Figure 5). 

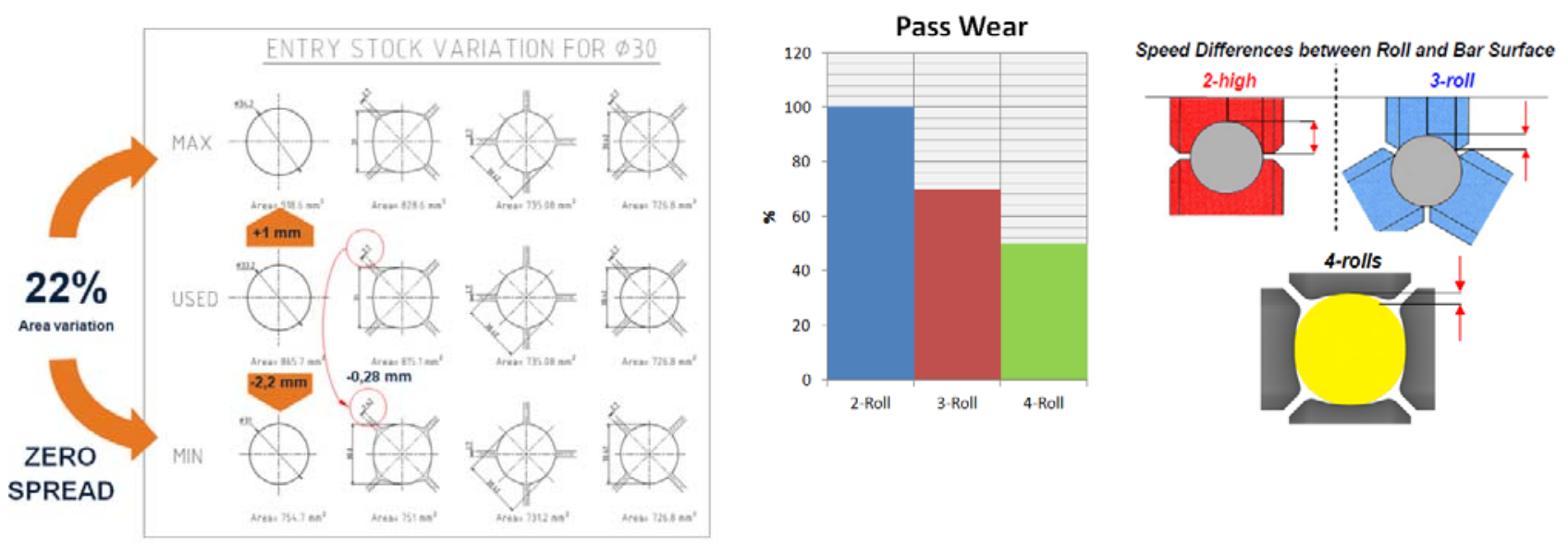

Figure 5. Entry area compensation in 4-roll module and pass wear reduction vs. 2- or 3-roll

The 4-rolls method allows a large gap adjustment and therefore also a wider freesizing rolling range. This is related to the higher percentage of the rolled material's outline in contact with the rolling rolls (constrained by contact with rolls) compared to that in the 3-roll method.

This pass design grants considerably larger free sizing range than those of the 3-roll system, as it can be appreciated from the below figure 6 .
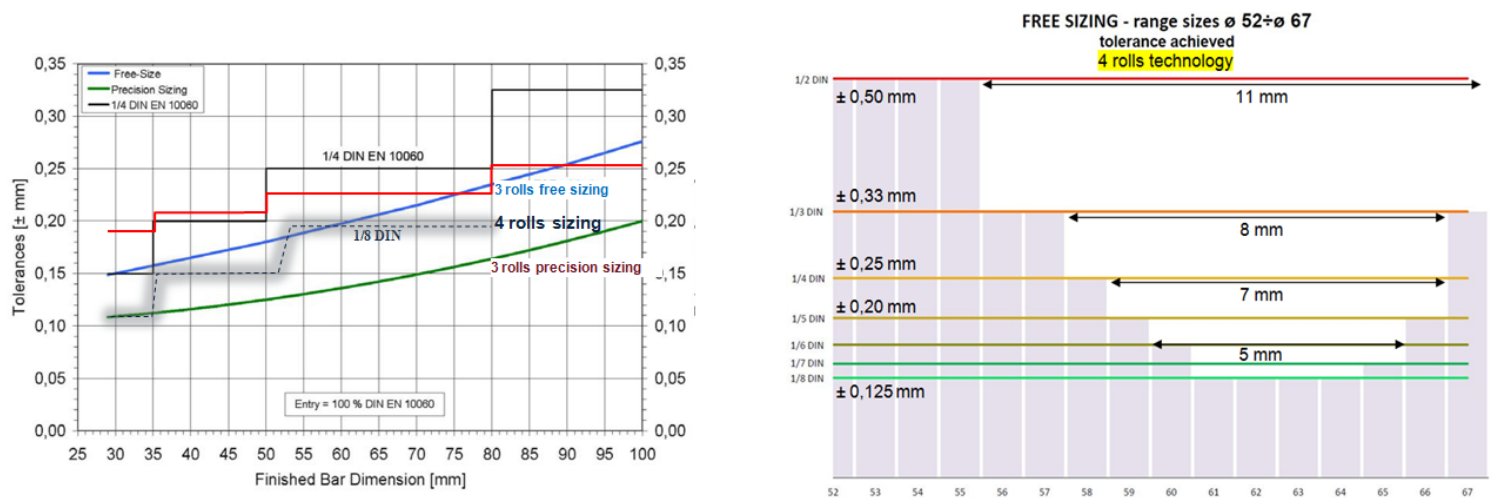

Figure 6. Free-sizing capacity with EVO 2+4-roll mill

The 4-roll finishing sequence can assure extremely accurate tolerance (up to 1/8 DIN) even when rolling in normal free-sizing mode (i.e. $4 \mathrm{~mm}$ range); 1/6 DIN can be still obtained when rolling with enlarged free-sizing range (i.e. $5 \mathrm{~mm}$ ) and even 1/4 DIN with extra-large free-sizing range $(7 \mathrm{~mm})$. An extreme application can be to roll still with $1 / 2$ DIN accuracy with a free-sizing range of up to $11 \mathrm{~mm}$.

The same flexibility and accuracy of use cannot be obtained by the 3-roll rolling method, due to the tri-lobed shape of the rolls. For this reason the 3-roll technology is normally conceived to work with a double type of pass sequence: one for the precision sizing rolling (up to 1/8 DIN) and one different for the free-sizing rolling. While working in the free-sizing mode, even if with a more narrow range compared to 4 roll, the obtained tolerance with 3-roll is immediately degraded.

As a basic comparison, while working with a free-sizing range of $5 \mathrm{~mm}$, the 4-roll sequence would allow to get $1 / 6$ DIN accuracy against the $1 / 2$ DIN of the 3-roll. The 4roll sequence can therefore grant precision sizing and wide range free-sizing together, while 3-roll cannot. 
The peculiar profile of the 4-roll rolls combined with the simplified pass schedule as described above, provides also a significant saving in the rolls inventory (up to minus $20 \%$ of number of rolls compared to a 3-roll application). Due to the lower number of stoppages for the change of rolling sequence, also the mill utilization index is drastically improved.

The consistency of the bar size under the different rolling conditions, gives a further immediate advantage to the bar producers that can obtain more length from the same billet (Figure 7).
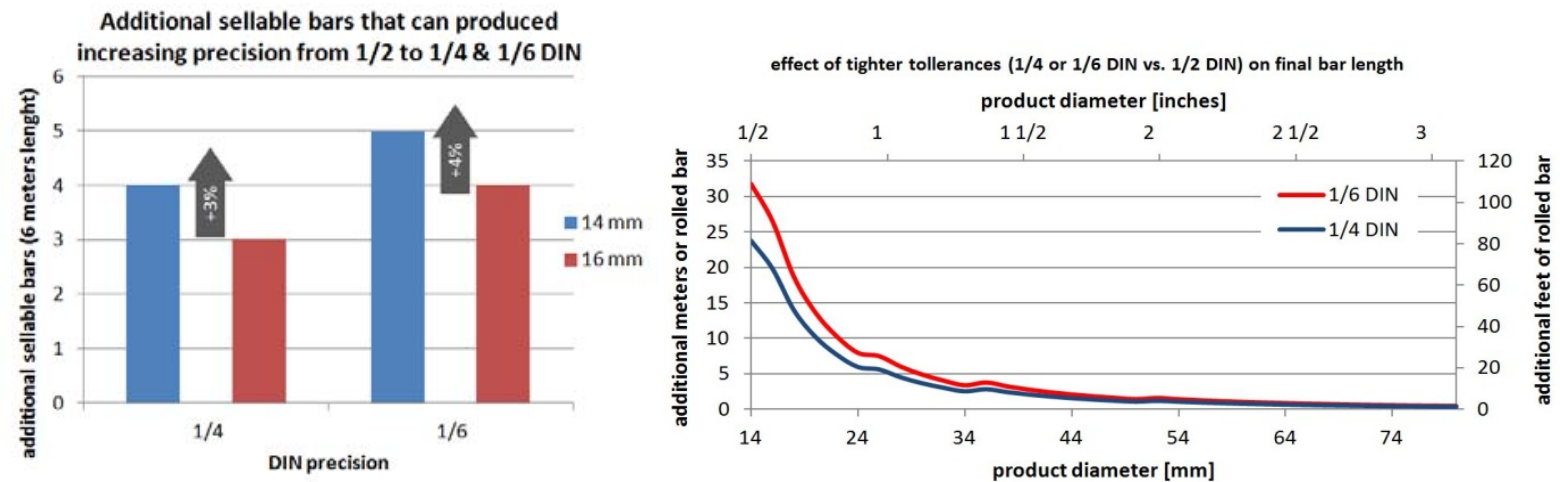

Figure 7. Additional saleable bars obtainable with precision rolling

It has also to be considered that the final shape obtained with the 2+4-roll rolling has very tight accuracy in terms of roundness. Instead the 3-roll rolling produces a threelobed rounds with abnormal roundness and center offset: this requires special measuring gauge to detect the size and can even create problems to the downstream automatic processes like peeling and drawing machines. Normally to remove the three-lobed effect from the rolled bar, a deep peeling has to be applied to the bars produced with 3-roll, thus wasting material and reducing the value of the rolled bars.

Considering the metallurgical implications of the reducing and sizing technologies, it can be stated that the two 2-roll stands employ an oval-round sequence with high reduction ratios, resulting in a deep and uniform grain refinement obtained from surface to core of the rolled stock, similarly to what a forging operation would obtain. A fine grain is beneficial to mechanical properties, and it also eases the downstream processes, such as drawing and spring bending, bringing a reduction of corresponding cost.

As grain tends to dynamically recrystallize in between deformation passes, it is important that the traveling time from the exit of the 2-roll group to the finishing passes be reduced to a minimum. This is accomplished by placing the 2- and 4-roll groups at a very short distance to one another.

The three compact 4-roll stand employs a round-round-round sequence with light reduction ratios, in order to obtain precise shape and tolerances. While lower strain tends to make the grain coarse, the 4-roll design can effectively limit this, by controlling the spread and uniformity of plastic strains.

Thanks to the extremely high control on the lateral spread achievable with 4-rolls method, all the deformation work is efficiently utilized to shape the section and to elongate the bar. By mean of finite element simulation is possible to determine the extent of the energy used to achieve the same reduction with different methods: compared to 3-rolls method, the 4-rolls offer a saving of approx $15 \%$ of the energy (kWh/tons). 
All the additional deformation work to recovery the unwanted lateral spread causes unnecessary heat increase in the rolled stock when rolling with 3-roll (Figure 8) where there are localized high levels of strain and consequent material overheating, resulting in a less controlled grain size (local coarse grain 6-7 ASTM) and in a less uniform metallurgical texture of the as-rolled product (variation up to 2-3 ASTM from surface to core).
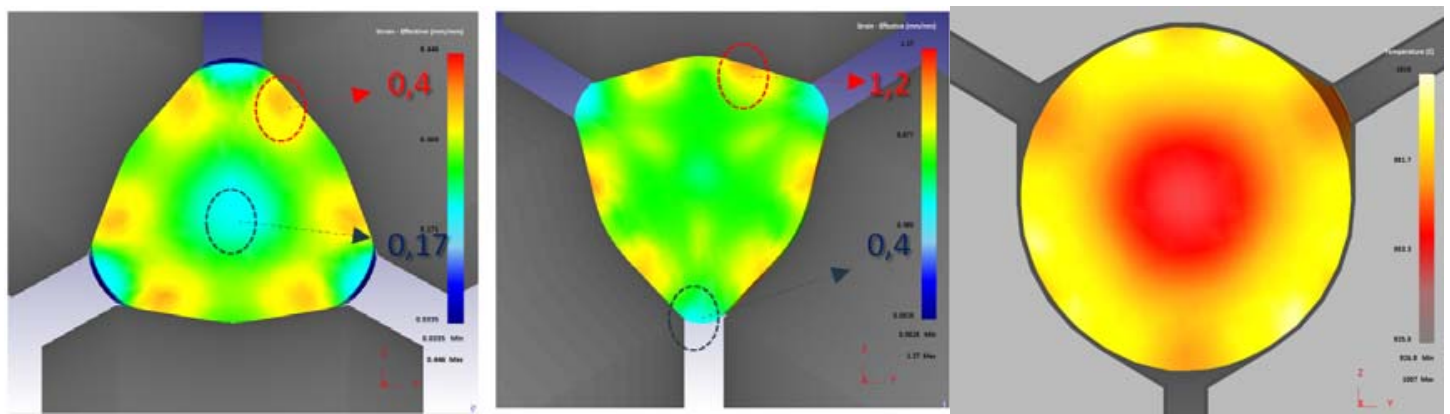

Figure 8. $\mathrm{t}$ High localized strain and temperature overheating with 3-roll

The uniform and zero-spread rolling of the 4-roll passes keep instead the strain and the temperature differences in the section at very low values (Figure 9), with large benefits for the obtainable grain size (> 8-9 ASTM) and for the metallurgical uniformity (variation of $<1$ ASTM from surface to core).
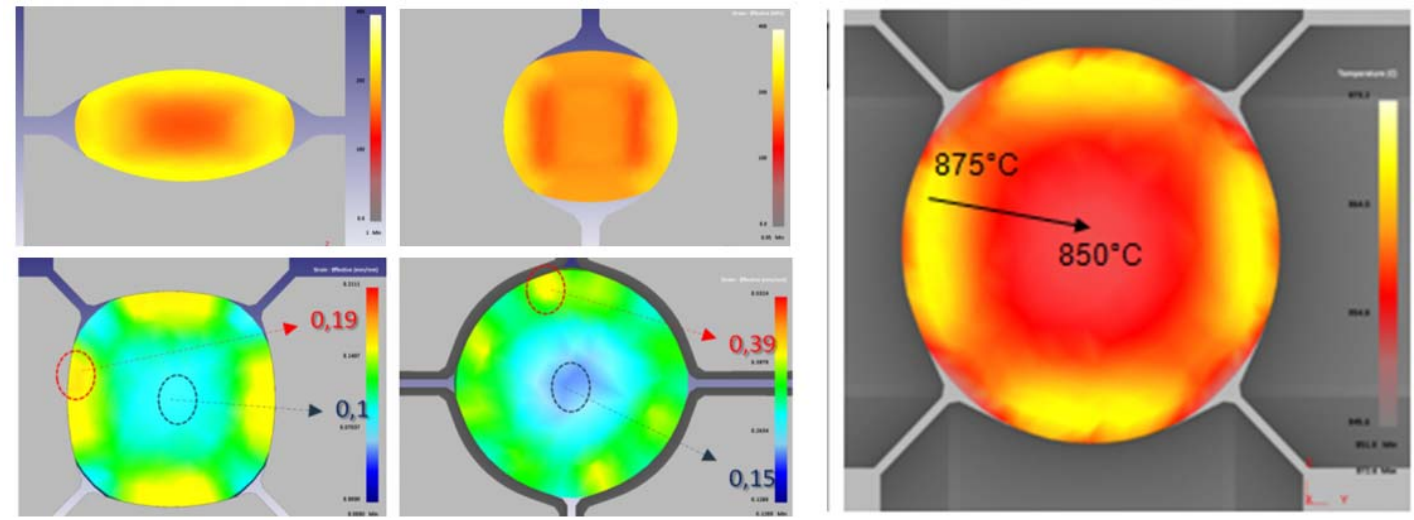

Figure 9. Uniform strain distribution and low temperature gradient with 2+4-roll sequence

The 4-roll technology allows higher $\%$ of reduction on the entry stock with a constantly uniform distribution of the rolling pressure along the $360^{\circ}$ of the bar circumference and with the resulting forces always balanced. This, combined with the very short inter-stand $(<400 \mathrm{~mm})$ distance in the 4-roll module, gives a stable twistfree process which allows to roll with simple anti-friction static guides (Figure 10).
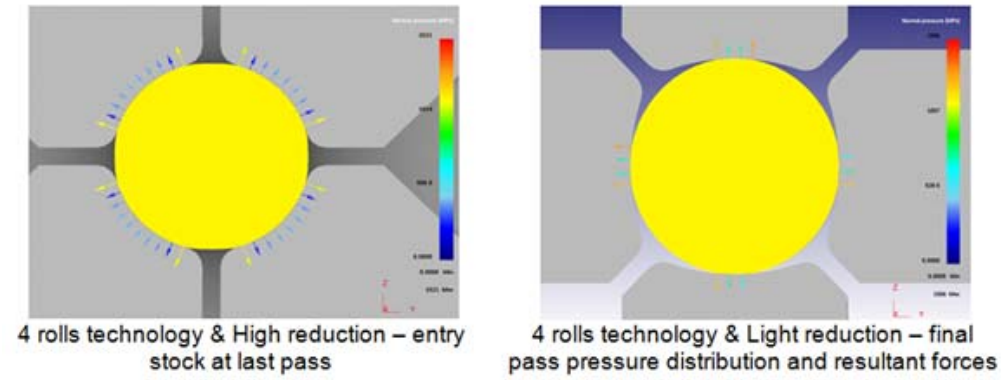

Figure 10. Balanced rolling forces 
The pressure distribution and the resulting forces are instead not balanced with the 3-roll technology (Figure 11) thus making the rolling a bit unstable and needing 3-roll stabilizing guides between the stands. Therefore there are additional parts to be set and maintained. The twist effect depends on the uneven distribution of the forming pressure on the rolled stock that generates resultant torque moment; high reduction rate and deviation of the entry stock from ideal symmetrical shape contribute to worsening this effect.

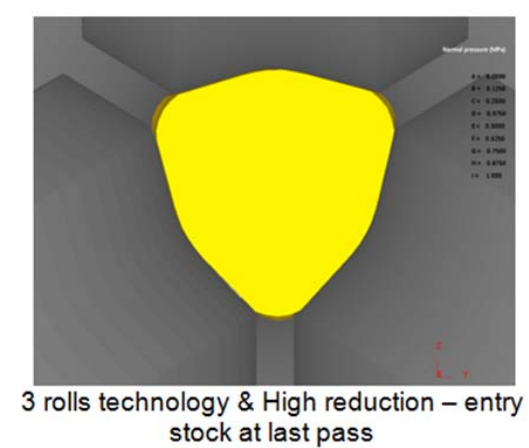

Figure 11. Unbalanced rolling forces in 3-roll

\section{HIGHLIGHTS OF EQUIPMENT DESIGN}

The EVO 2+4-roll Mill is composed by internal cartridges containing the rolls clamped within an holding external housing frame. The connection of the cartridge to the housing frame and the individual rolls gap adjustment, are actuated by hydraulic capsules (Figure 12) connecting by means of a T-locking head.

They grant high load capability and quick automatic compensation of the process variables, as necessary to enhance the advantages of the true-thermomechanical rolling combining the low temperature rolling with high reduction rates. Specifically the 2-roll stands can bear very high rolling loads to cope with the single-family rolling requirements, providing a uniform and deep plastic deformation to the material, while the 4-roll stands zero-spread rolling grants a repeatable and accurate shape control.

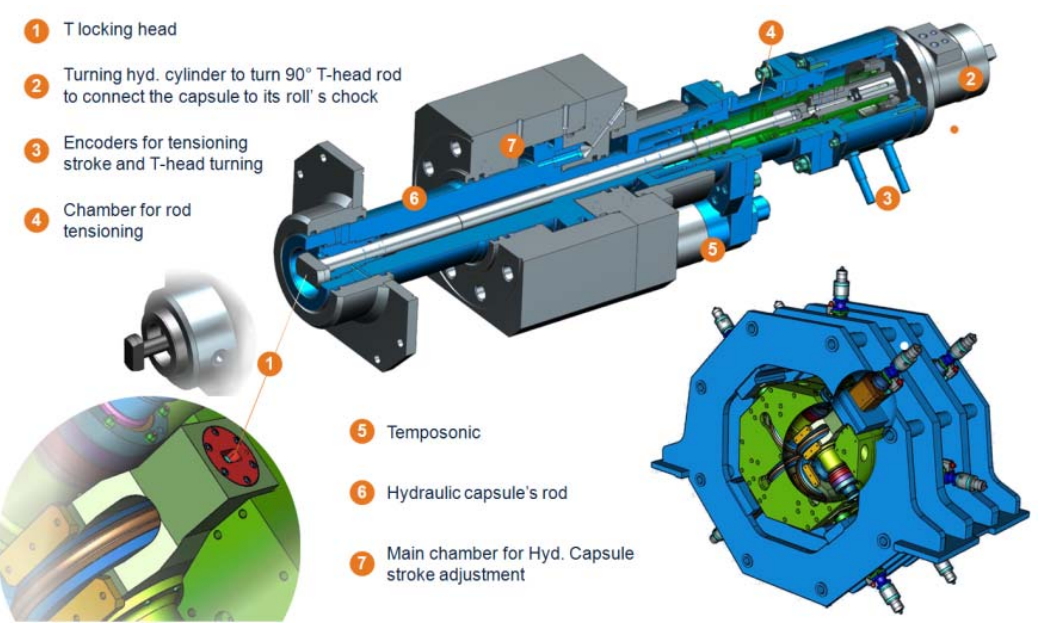

Figure 12. Cartridge and hydraulic capsule arrangement of 4-roll stand

Besides, as setup operations are completed, rolling may be immediately restarted obtaining the desired tolerance right from the first bar, without any trial bar. The 
capsules also provide an anti-jamming function in case of emergency to releases cobbled bars without damaging the parts. Likewise, temperature and vibrations sensors are provided in the group, so that variations of temperature and abnormal working conditions may be monitored along the whole bar and during production campaign.

All the above information are integrated in the Dimensional Control Module (DCM), along with the information of size, shape, temperature, stand stiffness (spring effect), gap, rolling force. Since larger or smaller entry sections, and lower or higher temperatures, respectively increase or reduce the separating forces, the DCM provides the necessary adjustment to the gap of each roll, in real time (Figure 13).

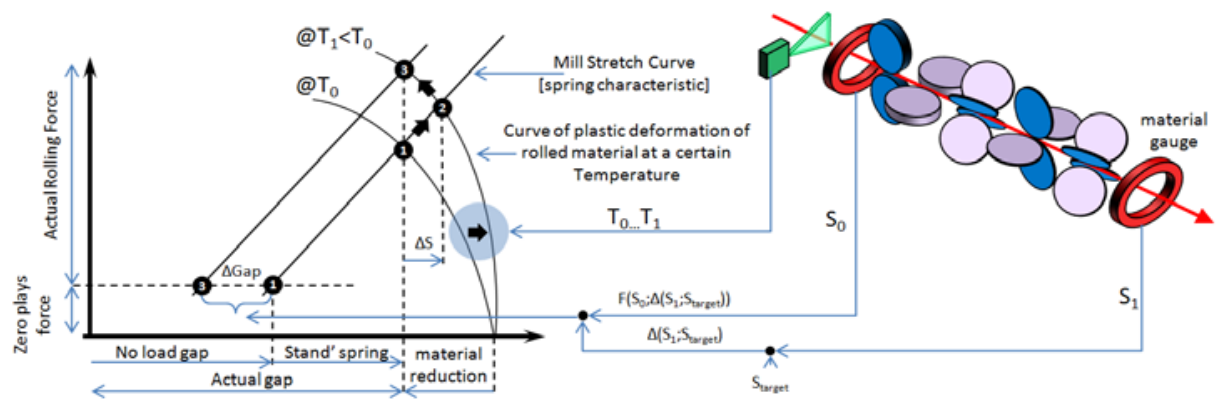

Figure 13. Real-time gap adjustment with DCM

The EVO 2+4-roll mill is conceived to have the drive transmission system completely detached from the rolling cartridge. The motion to the rolls are given by means of gear distribution chains for each couple of rolls, both for the 2-roll and the 4-roll stands. The specific split design for the 4-roll stand, meaning that there are two motors and independent distribution system for each of the 4-roll stands, allow to keep a lean design and an easiness of speed synchronization for the motors, not dissimilar from that used in high-speed finishing blocks.

The correct calculation of the inter-stand distances ensures a good stability of the rolling stock. Bar twisting must be avoided due to its associated risks of cobbles, tolerance deterioration, surface damage. Between the two 2-roll stands a roller guide is inserted to stabilize the position of the oval stock. At the exit pass of the second 2roll stand a static guide is employed to drive the round stock into the 4-roll group. Special friction-free static guides are employed between the 4-roll stands, where the small inter-stand distance and the balanced forces promote a very stable operation.

The setup operation of the EVO 2+4-roll mill is similar to that of a machine tool, that is automatic, easy and consistently repeatable over time. Stands and guides preparation are performed in the roll shop, in parallel to rolling. This leads to changing times of rolls and stands in accordance with modern operation practices.

The pack of the 2-roll and 4-roll cartridges can be extracted laterally and independently with an automatic device, then replaced with the new set of cartridges prepared offline for the next rolling campaign (Figure 14). The times for the stands change are very short thus granting the maximum time for the mill utilization.

The times for the main operational setup activities in the 2+4-roll mill are:

- in-line size change in free-sizing mode: 1 minute

- complete change of module (2-roll and 4-roll modules, together or independently) to new size family: 3 minutes

- offline disassembling of stands module and rolls change: 15 minutes 
TOP VIEW
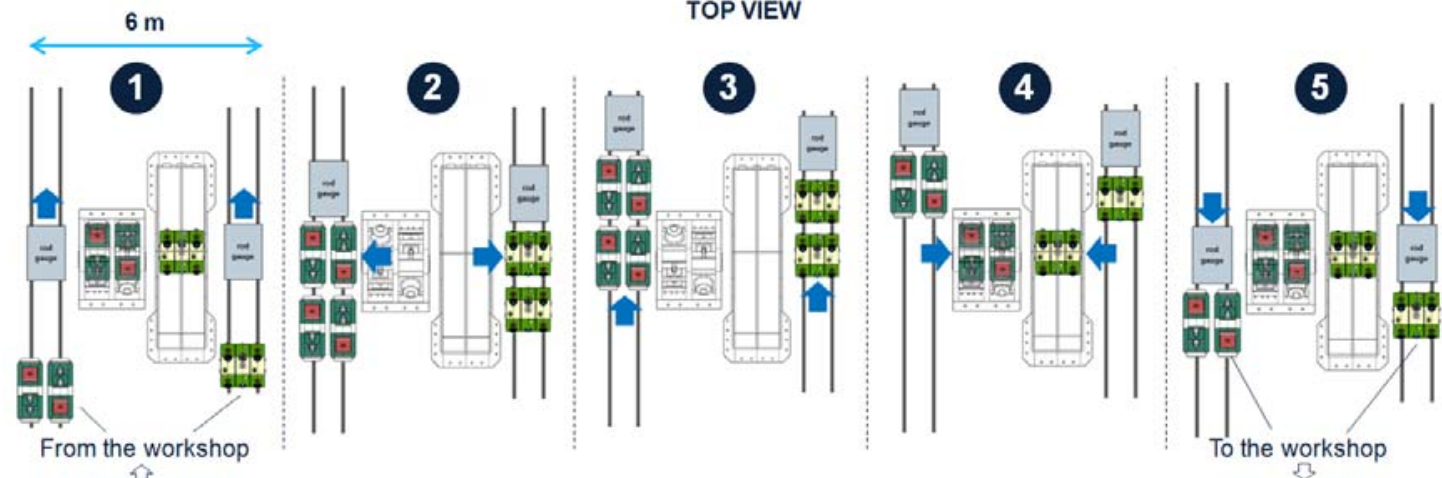

Figure 14. Change sequence of $2+4$-roll modules

\section{SUMMARY}

With the integration of 2- and 4-roll stands, the EVO 2+4-roll Reducing and Sizing Mill ensures very accurate dimensional tolerances, an excellent surface finishing and a precisely controlled metallurgical micro-structure.

A very wide range of sizes may be processed with a given diameter of profiled rolls, limiting the requirement of roll sets, with savings of both capital and operational expenditures. As operations of roll change and setup are reduced, the utilization and therefore productivity of the mill are greatly improved. Benefits are significant over traditional technologies.

\section{REFERENCES}

1 Primetals Technologies: Evolution of Bar Sizing, 84th AIKW Conference 2014

2 Kawasaki Steel Corporation: 4-Roll Size-Free Mill for Bar and Wire Rod

3 Morgan Construction Company: 2-Roll and 3-Roll Rolling

4 Tomoyasu Sakurai, Toshio Sakamaoto, Ryo Takeda: Development of High Dimensional Accuracy Smaller Diameter Wire Rods and Square Coils Manufactured by 4-Roll Mill, Kawasaki Steel Technical Report, 2002

5 Nippon Steel \& Sumikin Stainless Steel Corporation: Outline of Free Size Rolling for Hiraki Wire Rod and Bar Mill

6 US Patent US 6546777 B2 "Method and apparatus for reducing and sizing hot rolled ferrous products"

7 European Patent EP1315585B1 "Method for reducing and sizing hot rolled ferrous products" 